\title{
Spiderweb deformation induced by electrostatically charged insects
}

SUBJECT AREAS:

BIOMECHANICS

BIOINSPIRED MATERIALS

ENTOMOLOGY

COEVOLUTION

Received

28 February 2013

Accepted

14 June 2013

Published

4 July 2013

Correspondence and requests for materials should be addressed to

V.M.O.-J. (vortega@ unc.edu)
Victor Manuel Ortega-Jimenez' \& Robert Dudley ${ }^{1,2}$

'Department of Integrative Biology, University of California, Berkeley, CA 94720 USA, ${ }^{2}$ Smithsonian Tropical Research Institute, P.O. Box 2072, Balboa, Republic of Panama.

Capture success of spider webs has been associated with their microstructure, ornamentation, and wind-induced vibrations. Indirect evidence suggests that statically charged objects can attract silk thread, but web deformations induced by charged insects have not yet been described. Here, we show under laboratory conditions that electrostatically charged honeybees, green bottle flies, fruit flies, aphids, and also water drops falling near webs of cross-spiders (Araneus diadematus) induce rapid thread deformation that enhances the likelihood of physical contact, and thus of prey capture.

T he capture effectiveness of orb-weaver webs has been attributed to mechanical ${ }^{1,2}$, hygroscopic ${ }^{3}$, and adhesive $^{4}$ characteristics of the constituent silk, as well as to architectural features ${ }^{2,5}$, ornamentation ${ }^{6}$, and windinduced distortions of the entire structure ${ }^{7}$. Despite suggestions that web deformation can be induced by the human finger ${ }^{8}$, that insects can be easily charged ${ }^{9-11}$, and that foraging honeybees can acquire charge sufficient to detach pollen from flowers ${ }^{12}$, the effects of electrical charge on spider orb webs are unknown. Here, we evaluated the deformation responses of spider webs as induced by statically charged insects and water drops.

\section{Results}

Video sequences of positively charged insects and water droplets falling towards a grounded orb web reveal rapid and substantial web attraction (Supplementary Movie S1 online). Radial and particularly spiral silk threads are quickly attracted to the electrified bodies. By contrast, control trials using uncharged insects (honeybees, $\mathrm{N}=3$, 15 trials; bottle flies, $\mathrm{N}=3,11$ trials; fruit flies, $\mathrm{N}=4,20$ trials; aphids, $\mathrm{N}=2,6$ trials) and drops $(\mathrm{N}=10)$ show no such deformation (Supplementary Movie S2 online).

It is important to note that $\sim 30 \%$ and $\sim 10 \%$ of charging experiments with insect bodies and water drops, respectively, yielded no evident web deformation but were nonetheless included in analyses. For these cases, video recordings indicated that either the distance between the falling body and the spiral thread was $>\sim 2$ body lengths (suggesting much reduced electrical attraction following the inverse square law) or because the body hit directly a radial thread with minimal extensibility (Supplementary Movie S3 online).

Maximum deformations of spiral silk threads from their resting positions ranged from about 1-2 $\mathrm{mm}$, depending on the size of the charged test object (Table 1); length-normalized deformations ranged from about $0.2-0.7$ (Table 1, Fig. 1). Average thread speeds during such movements were on the order of $0.7-1.9 \mathrm{~m} / \mathrm{s}$ (see Table 1). Spiral threads contacted by a falling water drop could also acquire its positive charge, which resulted in electrical repulsion if an additional drop then fell immediately in its vicinity (Supplementary Movie S4 online).

No significant differences were found in the magnitude of induced web deformation among individual honeybees $(\mathrm{F}=0.83, \mathrm{df}=2,18, \mathrm{P}=0.45)$, green bottle flies $(\mathrm{F}=0.58, \mathrm{df}=2,20, \mathrm{P}=0.57)$, fruit flies $(\mathrm{F}=$ $2.3, \mathrm{df}=3,28, \mathrm{P}=0.1$ ), aphids (Wilcoxon's rank-sum test $\mathrm{W}=12.5 \mathrm{P}=0.62$ ), and the two sizes of droplets (Wilcoxon's rank-sum test $\mathrm{W}=63.5, \mathrm{P}=0.57$ ); (Supplementary Table $\mathrm{S} 1$ online). Voltages among individual insects were similar for each of the study species (honeybees: $\mathrm{F}=0.23, \mathrm{df}=2,27, \mathrm{P}=0.8$; green bottle flies: $\mathrm{F}=$ $3.05, \mathrm{df}=2,27, \mathrm{P}=0.06$; fruit flies: $\mathrm{F}=0.3, \mathrm{df}=2,12, \mathrm{P}=0.74$ ) (Supplementary Table S2 online)(Table 1). However, voltage of large water drops was significantly higher than that of small drops $\left(\mathrm{t}_{38}=7.2, \mathrm{P}<0.0001\right)$.

\section{Discussion}

Electrostatic forces have been previously proposed to play an important role in silk adhesion ${ }^{14-16}$, although experimental evidence indicates that only non-electrostatic adhesive properties pertain to cribellar silk ${ }^{15}$. Our experiments show clearly that positively charged insect bodies induce rapid attraction of silk threads in the webs of cross-spiders, indirectly supporting a prior hypothesis that static charges of insects increase the prey capture 
Table 1 Morphological data and electrical properties for charged test objects, and associated web deformations and average speeds of deformation (mean \pm S.D. [sample size N]). Body mass, body length and voltage were measured repeatedly on the same insect ( 3 individuals $\times 10$ repetitions, except for fruit flies with 3 individuals $\times 5$ repetitions) or water drop (20 large drops and 20 small drops). Web deformations were measured on different individual insects (honeybees, $N=3$; bottle flies, $N=3$ fruit flies, $N=4$; aphids, $N=2$ ) and drops. Voltages of charged aphids could not be measured relative to background noise of the sensor. See text for details

\begin{tabular}{lccccccc} 
Test object & Mass $(\mathrm{mg})$ & Length $(\mathrm{mm})$ & Voltage $(\mathrm{kV})$ & Charge $(\mathrm{pC})$ & $\begin{array}{c}\text { Deformation } \\
(\mathrm{mm})\end{array}$ & $\begin{array}{c}\text { Length-normalized } \\
\text { web deformation }\end{array}$ & $\begin{array}{c}\text { Deformation } \\
\text { speed }\left(\mathrm{ms}^{-1}\right)\end{array}$ \\
\hline Honeybee & $86 \pm 7(3)$ & $11.7 \pm 0.4(3)$ & $0.5 \pm 0.2(30)$ & $180 \pm 61(30)$ & $2 \pm 2(21)$ & $0.2 \pm 0.2(21)$ & $2 \pm 1(18)$ \\
Bottle fly & $19 \pm 3(3)$ & $7.9 \pm 0.4(3)$ & $0.3 \pm 0.1(30)$ & $94 \pm 18(30)$ & $1 \pm 1(23)$ & $0.1 \pm 0.1(23)$ & $1.0 \pm 0.3(19)$ \\
Fruit fly & $2.5 \pm 0.4(3)$ & $3.1 \pm 0.2(3)$ & $0.06 \pm 0.02(15)$ & $22 \pm 7(15)$ & $1 \pm 1(32)$ & $0.2 \pm 0.2(32)$ & $1 \pm 1(24)$ \\
Aphid & $0.6 \pm 0.2(3)$ & $1.8 \pm 0.3(3)$ & - & - & $1 \pm 1(9)$ & $0.5 \pm 0.4(9)$ & $0.8 \pm 0.4(7)$ \\
Large drop & $5 \pm 1(3)$ & $2.2 \pm 0.1(3)$ & $0.22 \pm 0.04(20)$ & $73 \pm 14(20)$ & $1 \pm 1(11)$ & $0.5 \pm 0.2(11)$ & $0.8 \pm 0.4(10)$ \\
Small drop & $1.6 \pm 0.2(3)$ & $1.4 \pm 0.1(3)$ & $0.14 \pm 0.03(20)$ & $46 \pm 9(20)$ & $1.0 \pm 0.4(10)$ & $0.7 \pm 0.3(10)$ & $0.9 \pm 0.4(9)$ \\
\hline
\end{tabular}

success of orb-webs ${ }^{15}$. Risk of capture for a free-flying insect may correspondingly be enhanced given that the induced deformations observed here are comparable to the average mesh spacing for crossspider webs $^{17}(\sim 2 \mathrm{~mm})$. Observed deformations also suggest that greater charges and associated web displacements may characterize larger insects, and are more likely to induce deformation of multiple radial and spiral threads (Table 1). By contrast, length-normalized deformations suggest non differences among insects (Figure 2; see also Table 1 and supplementary Table S1 online). The greater charge typically accumulated on larger test insects (see Table 1) reflects its direct dependence on both electric potential (as induced here by the Van de Graaff generator) and their capacitance. Electrostatic charge acquired by insects in free flight will similarly reflect these factors, but charge will principally be acquired through interaction between the flapping wings and the surrounding air, along with particular atmospheric conditions that promote charge accumulation (e.g., lower relative humidity).

The substantial variance in web deformation data (Table 1) likely reflects the effects of variable body position and orientation with respect to silk threads, as must characterize prey captured by

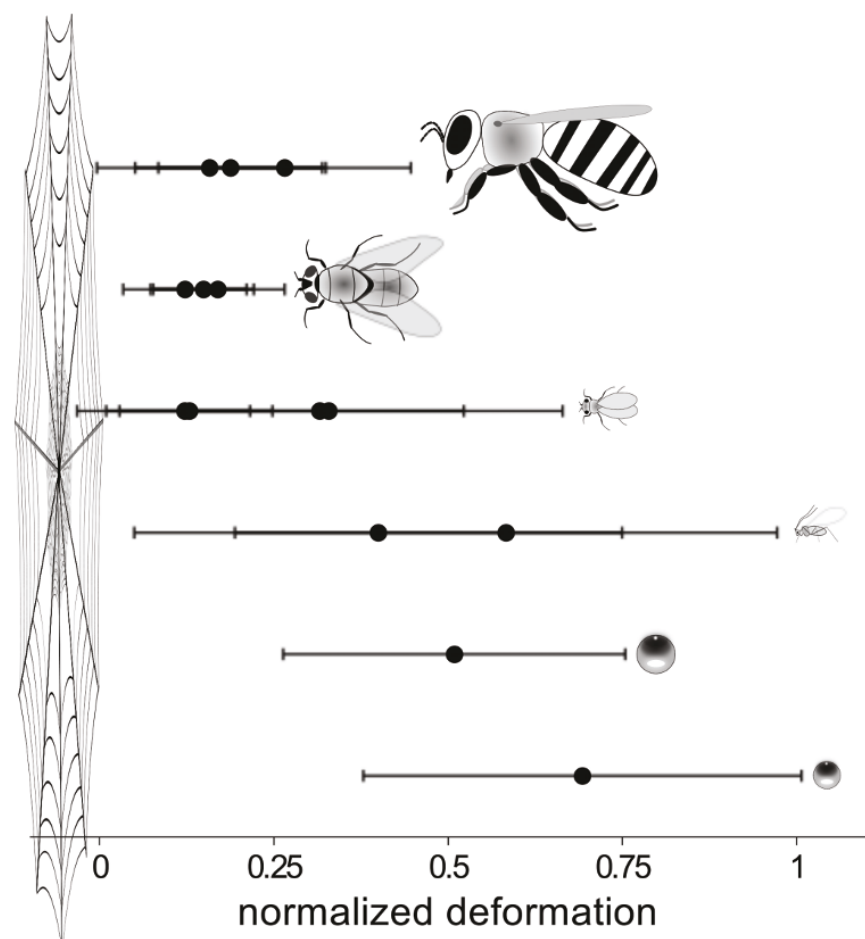

Figure $1 \mid$ Normalized web deformation produced by statically charged insects and water drops. Black dots represent the average value of each sampled individual. Error bars represent 1 s.d. spiderwebs in nature. Because electrostatic force varies with the inverse square of distance, substantial differences in thread deformation for charged objects passing through the web can be expected. Deformation data presented here, moreover, refer only to twodimensional motions and will systematically underestimate total thread displacement. The extensibility of web spiral thread is higher than that for radial thread ${ }^{1}$, and we observed qualitative differences in thread deformation according to the position of charged fruit flies falling relative to these two distinct web elements (Supplementary Movie S5 online). It is also well known that spiral threads of ecribellate spiders are more extensible than those of cribellate species. A recent computational study found that increased elasticity of spiral threads reduces the energy absorption and dissipation of the entire web, but in contrast is mostly unchanged for individual spiral threads, despite reduction in strength ${ }^{18}$. These results suggest that comparable capture effectiveness for spiral threads can be obtained with high elasticity and low strength (and that require lower energetic investment to produce). Accordingly, charged insects or small particles may elicit greater deformations from spiral threads of ecribellate taxa.
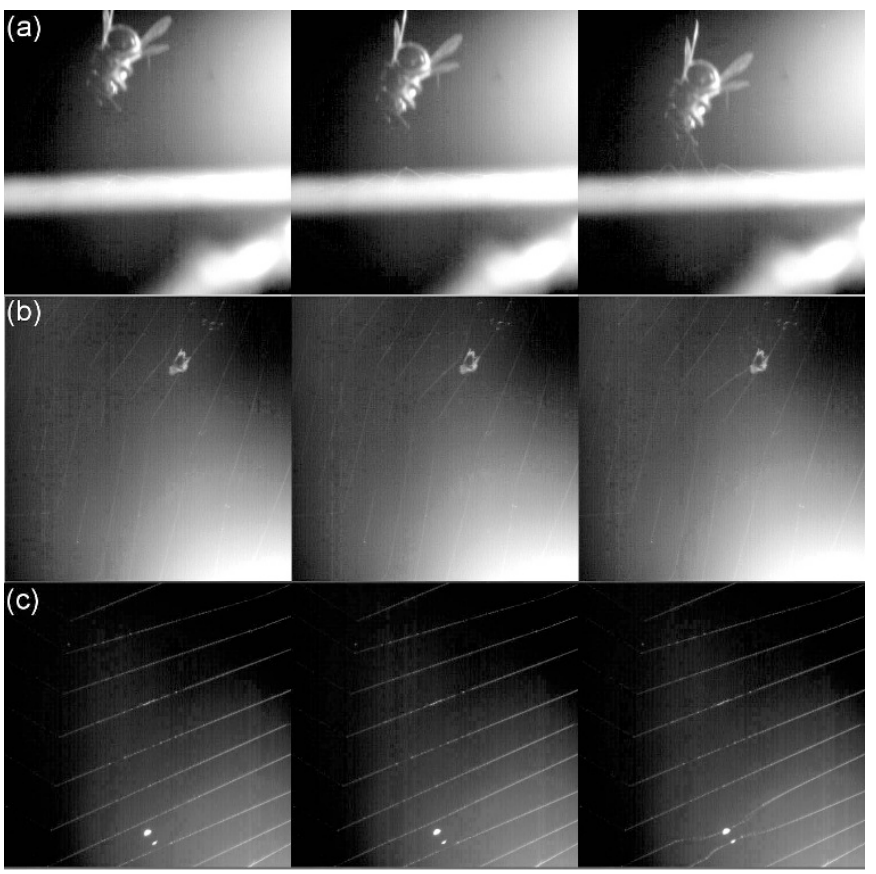

Figure $2 \mid$ Web deformation produced by a charged honeybee (a), a fruitfly (b) and a water drop (c). Images are three sequential video frames (filming speed: 1500 frames $\mathrm{s}^{-1}$ ). Bee, fruitfly and drop size are $12 \mathrm{~mm}$, $3 \mathrm{~mm}$ and $1.5 \mathrm{~mm}$, respectively. Image gamma was increased to 1.5. 
Insects can easily acquire electrostatic charge by walking over charged surfaces or by flying in an airstream of charged particles ${ }^{9-11}$. Honeybee workers ${ }^{11}$ during wintertime conditions can acquire a positive charge of up to $537 \mathrm{pC}$, whereas honeybees those in nonwinter weather can reach up to $200 \mathrm{pC}$, values comparable to those used here experimentally (Table 1). Positively charged bumblebees can even detect floral electric fields, which ability enhances their foraging success ${ }^{19}$. Despite the well-recognized role of electrostatic charge in pollination ${ }^{12}$, the charge magnitudes on insects in nature are largely unknown, but are expected to be higher in low humidity and in dusty conditions.

Spider-orb webs are aerial traps specialized to catch flying insects of different sizes, and even occasionally birds ${ }^{20}$. Mechanical properties of the web silk dissipate the kinetic energy and impulse acting to the web produced during insect impact ${ }^{1}$. The stickiness and elasticity of ecribellate threads are mediated by their water coating ${ }^{3,4}$, which has a $\sim 80$ times higher value for relative permittivity than air $^{21}$. Wet silk threads may thus be more easily polarized by an electrostatic field than are dry threads. In calm weather, air contains predominantly positive ions, in contrast to vegetation that is typically negatively charged ${ }^{22}$. Although charges of spider webs under natural conditions have never been reported, accumulation of negative charge may result in even greater deformation in response to positively charged insects. Charge accumulation may also increase deposition rates of electrically charged particles floating in the air, such as pollen and fungal spores, which are actually consumed by juvenile cross-spiders ${ }^{23}$. However, adverse dust deposition and associated web degradation may influence daily patterns of web reconstruction ${ }^{24}$, to which end charge accumulation may be a previously unrecognized contributing factor. Charged rain drops may

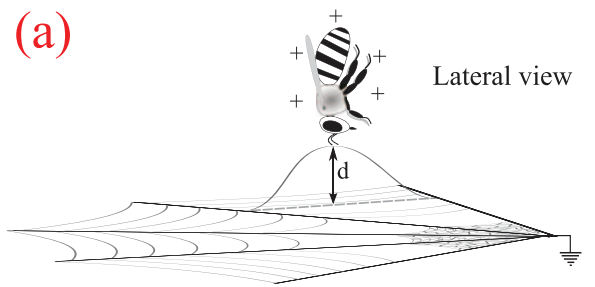

(d) Van de Graaf generator +

(b)
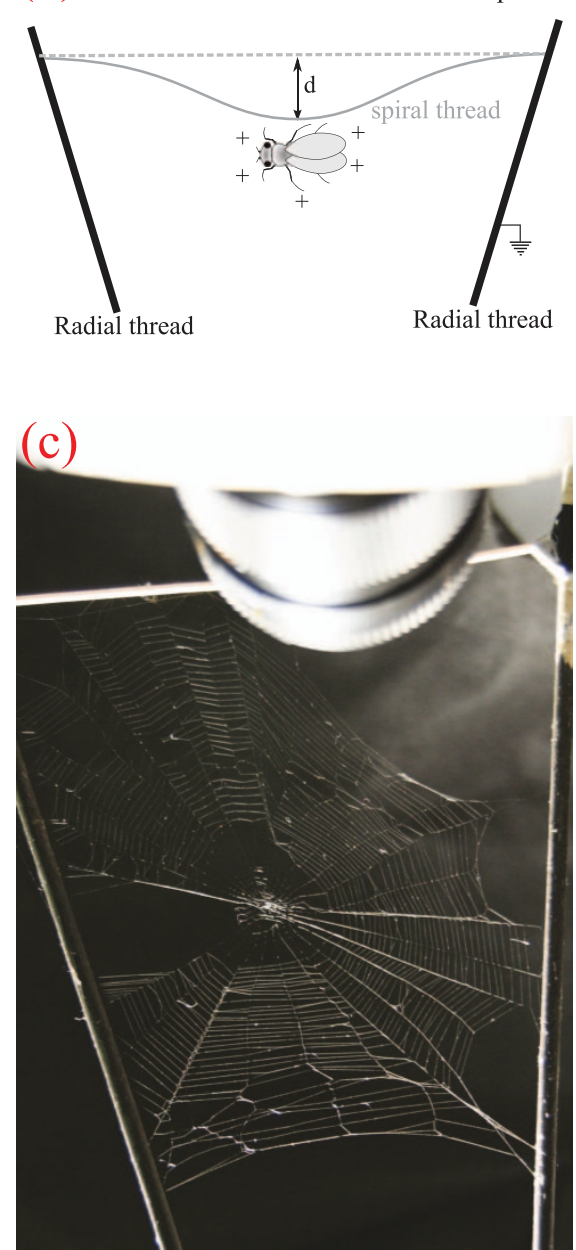
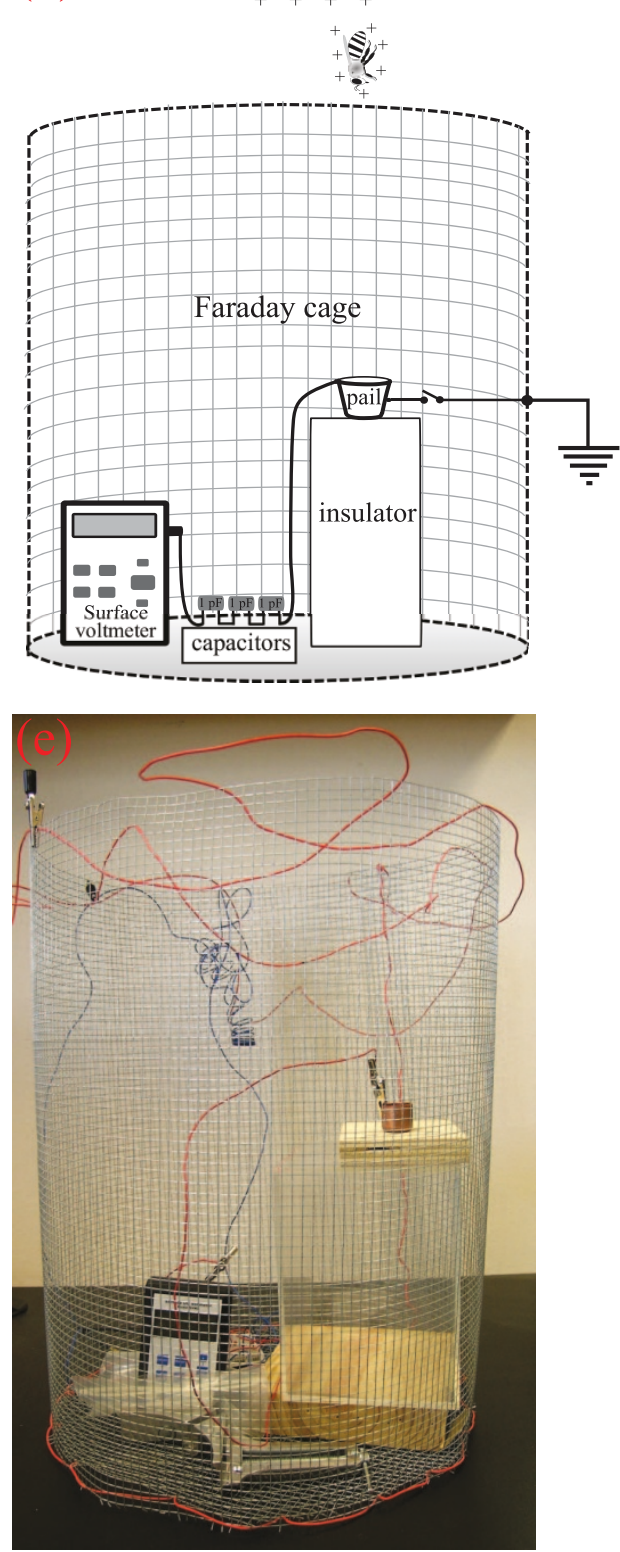

Figure 3 Experimental configurations used in measurements. (a) vertical web deformation induced by charged honeybees and bottle flies; (b) lateral thread deformation of charged fruit flies, aphids, and water drops; (c) Wood frame used to fix the spider web during filming; (d) electrical charge of experimental objects; and (e) apparatus used for voltage measurements (capacitors are located behind the voltmeter). Note that the typical orientation of falling insects in (a) and (b) was not regularly aligned as indicated. 
also induce web damage or adhesion of adjacent threads; field measurements during spring rainfalls indicate both positive and negative charges on rain with values of up to $100 \mathrm{pC}^{25}$.

In conclusion, we have experimentally demonstrated that electrostatically charged insects and water drops can induce rapid and comparably sized deformations in threads of the cross-spider's orb web. Such deformations likely increase the risk of capture for freeflying prey.

\section{Methods}

Orb webs of the cross-spider (Araneus diadematus), honeybees (Apis mellifera), green bottle flies (Lucilia sericata), fruit flies (Drosophila melanogaster) and aphids (Aphidoidea) were collected on the UC-Berkeley campus. Each spiderweb was collected via superposition onto a trapezoidal frame of wooden sticks, with radial and frame threads then being wrapped further to stabilize the web.

Freshly killed insects were positively charged for $\sim 3 \mathrm{~s}$ using a portable Van de Graaff generator (Unitech Toys, Foster City, CA). We tested only positive charge because data for foraging honeybees indicate this condition for $90 \%$ of individuals ${ }^{10}$. Insects were placed on a small section of aluminum foil $(1.5 \times 3 \mathrm{~mm})$ connected to the copper tip of the charge generator. Water drops were generated using a syringe and needles of two internal diameters (small: $0.16 \mathrm{~mm}$; large: $0.5 \mathrm{~mm}$ ), and were charged by placing the needle in contact for $\sim 3 \mathrm{~s}$ with the generator's tip.

Immediately after being charged, sampled individuals were dropped, from heights of $25-35 \mathrm{~cm}$, onto a horizontally oriented orb web mounted on a grounded wooden frame. These heights correspond to vertical descent speeds of $2.2-2.6 \mathrm{~m} / \mathrm{s}$ at the plane of the spider web. Neutrally charged insects and drops were also used in control experiments by placing them for $\sim 3 \mathrm{~s}$ on a grounded aluminum sheet prior to dropping. Relative humidity ranged between $47 \%$ and $50 \%$, and room temperature varied from $23-26^{\circ} \mathrm{C}$ during experiments.

Voltage and charge measurements. The sensor disk of a surface voltmeter (Model SVM2, AlphaLab Inc.) was electrically connected to three $1 \mathrm{pF}$ capacitors connected in series and then to a small copper pail (Fig. 3). A grounded cylindrical Faraday cage $(30 \mathrm{~cm}$ diameter $\times 45 \mathrm{~cm})$ was used to surround the entire test apparatus. Prior each measurement, the pail was grounded and the voltmeter was reset. Immediately after the pail was disconnected from ground, a test insect or drop was positively charged above the cage and was then dropped into the pail, yielding a peak voltage measurement at the surface voltmeter. The total accumulated charge was then estimated by multiplying this measured voltage by the total capacitance of the serially connected voltmeter ( $3 \mathrm{nF}$ ) and the three $1 \mathrm{pF}$ capacitors (Fig. 3).

Filming. Free falls of charged and uncharged insects and drops were filmed at 1500 2000 frames/s (Phantom v10, Vision Research, Wayne, NJ, USA; X-PRI, AOS Technologies AG, Baden Daettwil, Switzerland). Lateral recordings at the level of the spider web were performed for honeybees and bottle flies, and views from above were recorded for free falls of fruit flies, aphids and water drops. Web displacements were then recorded multiple times for each sampled insect (see Supplementary Table S1 for details). Video sequences were then digitized ${ }^{13}$, using for scale an absolute reference length recorded in the plane of view. Maximum vertical and lateral thread displacements (for lateral and vertical camera views, respectively), were then measured for each falling insect and water drop (see Fig. 3). For falling fruit flies, aphids, and water drops, lateral web displacements were made on two nearby spiral silk strands, which were assumed to be independent of one another. An average deformation speed was calculated by dividing total thread displacement by duration of measurement for those sequences characterized by obvious deformations.

Statistics. One-way ANOVA was used to test for significant differences in web deformation and maximum voltages among individuals in the separate groups of honeybees, bottle flies, and fruitflies. Wilcoxon's rank-sum test was used to test for deformation differences among sampled aphids and water drop sizes. Student's t-tests were used to compare voltages between the two sizes of drops.

1. Köhler, T. \& Vollrath, F. Thread biomechanics in the two orb-weaving spiders Araneus diadematus (Araneae, Araneidae) and Uloboris walckenaerius (Araneae, Uloboridae). J. Exp. Zool. 271, 1-17 (1995).

2. Cranford, S. W., Tarakanova, A., Pugno, N. M. \& Buehler, M. J. Nonlinear material behaviour of spider silk yields robust webs. Nature 482, 72-76 (2012).

3. Edmonds, D. \& Vollrath, F. The contribution of atmospheric water vapour to the formation and efficiency of a spider's capture web. Proc. Roy. Soc. London 248, 45-148 (1992).

4. Vollrath, F., Fairbrother, W. J., Williams, R. J. P., Tillinghast, E. K., Bernstein, D. T., Gallagher, K. S. \& Townley, M. A. Compounds in the droplets of the orb spider's viscid spiral. Nature 345, 526-528 (1990).
5. Lin, L. H., Edmonds, D. T. \& Vollrath, F. Structural engineering of an orb-spider's web. Nature 373, 146-148 (1995).

6. Craig, C. L. \& Bernard, G. D. Insect attraction to ultraviolet reflecting spider webs and web decorations. Ecology 71, 616-623 (1990).

7. Craig, C. L., Okubo, A. \& Andreasen, V. Effect of spider orb web and insect oscillations on prey interception. J. Theor. Biol. 115, 201-211 (1985).

8. Coddington, J. A., Chanzy, H. D., Jackson, C. L., Raty, G. \& Gardner, K. H. The unique ribbon morphology of the major ampullate silk of spiders from the genus Loxosceles (Recluse spiders). Biomacromolecules 3, 5-8 (2002).

9. Edwards, D. K. Electrostatic charges on insects due to contact with different substrates. Can. J. Zool. 40, 579-584 (1962).

10. Mcgonigle, D. F., Jackson, Ch. W. \& Davidson, J. L. Triboelectrification of houseflies (Musca domestica L.) walking on synthetic dielectric surfaces. J. Electrost. 54, 167-177 (2002).

11. Colin, M. E., Richard, D. \& Chauzy, S. Measurement of electric charges carried by bees: evidence of biological variations. J. Bioelectricity 10, 17-32 (1991).

12. Vaknin, Y., Gan-Mor, S., Bechar, A., Ronen, B. \& Eisikowitch, D. The role of electrostatic forces in pollination. Plant Syst. Evol. 222, 133-142 (2000).

13. Hedrick, T. L. Software techniques for two- and three- dimensional kinematic measurements of biological and biomimetic systems. Bioinspir. Biomim. 3, 034001 (2008).

14. Opell, B. D. What forces are responsible for the stickiness of spider cribellar threads? J. Exp. Zool. 265, 469-476 (1993).

15. Peters, H. M. The spinning apparatus of Uloboridae in relation to the structure and construction of capture threads (Arachnida, Araneida). Zoomorphology 104, 96-104 (1984).

16. Opell, B. D. Do static electric forces contribute to the stickiness of a spider's cribellar prey capture threads? J. Exp. Zool. 273, 186-189 (1995).

17. Vollrath, F., Downes, M. \& Krakow, S. Design variability in web-geometry of an orb-weaving spider. Physiol. Behav. 62, 735-743 (1997).

18. Tarakanova, A. \& Buehler, M. J. The role of capture spiral silk properties in the diversification of orb webs. J. R. Soc Interface 9, 3240-3248 (2012).

19. Clarke, D., Whitney, H., Sutton, G. \& Robert, D. Detection and Learning of Floral Electric Fields by Bumblebees. Science 340, 66-69 (2013).

20. Brooks, D. M. Birds caught in spider webs: a synthesis of patterns. Wils. J. Ornithol. 124, 345-353 (2012).

21. Yu, L., Hu, X., Kaplan, D. \& Cebe, P. Dielectric relaxation spectroscopy of hydrated and dehydrated silk fibroin cast from aqueous solution. Biomacromolecules 11, 2766-2775 (2010).

22. Bowker, G. E. \& Crenshaw, H. C. Electrostatic forces in wind-pollination? Part 2: Simulations of pollen capture. Atmosph. Environ. 41, 1596-1603 (2007).

23. Smith, R. R. \& Mommsen, T. P. Pollen feeding in an orbweaving spider. Science 226, 1330-1333 (1984).

24. Opell, B. D. Economics of spider orb-webs: the benefits of producing adhesive capture thread and of recycling silk. Funct. Ecol. 12, 613-624 (1998).

25. Chauzy, S. \& Despiau, S. Rainfall rate and electric charge and size of raindrops of six spring showers. J. Atmos. Sci. 37, 1619-1627 (1980).

\section{Acknowledgments}

We thank members of the Dudley flight mechanics lab and Daniel Hernández-Cruz for their valuable comments and suggestions, and also Sarahi Arriaga-Ramirez for helping us with the apparatus used to measure charge. Victor Ortega was supported a grant from the University of California MEXUS-CONACYT program.

\section{Author contributions}

Experimental design, analysis and interpretation of results, and writing of the manuscript were made by V.M.O. and R.D. The experiments were performed by V.M.O.

\section{Additional information}

Supplementary information accompanies this paper at http://www.nature.com/ scientificreports

Competing financial interests: The authors declare no competing financial interests.

How to cite this article: Ortega-Jimenez, V.M. \& Dudley, R. Spiderweb deformation induced by electrostatically charged insects. Sci. Rep. 3, 2108; DOI:10.1038/srep02108 (2013).

This work is licensed under a Creative Commons AttributionNonCommercial-ShareAlike 3.0 Unported license. To view a copy of this license, visit http://creativecommons.org/licenses/by-nc-sa/3.0 